\title{
Familial dentinogenesis imperfecta, blue sclerae, and wormian bones without fractures: another type of osteogenesis imperfecta?
}

\author{
P BEIGHTON \\ From the Department of Human Genetics, Medical School and Groote Schuur Hospital, \\ University of Cape Town, Observatory 7925, South Africa
}

\begin{abstract}
SUMMARY A unique connective tissue disorder characterised by the triad of dentinogenesis imperfecta, blue sclerae, and multiple wormian bones has been identified in 20 members of three generations of a large kindred of mixed ancestry in South Africa.

The skeletons of affected subjects were moderately osteoporotic but, apart from minimal bowing of the femora and some vertebral flattening in late adulthood, this abnormality produced no untoward sequelae. Bone fragility was present in one young male, while a mother and her daughter had deafness of uncertain relationship with the primary disorder. Dental discolouration and a liability to caries were the only important complications.

The condition is best regarded as yet another variety of osteogenesis imperfecta. It is inherited as an autosomal dominant trait with relatively consistent phenotypic expression.
\end{abstract}

The term 'dentinogenesis imperfecta' (DI) pertains to teeth in which defective dentine produces a yellowbrown discolouration and a tendency to caries. This abnormality may be inherited in isolation as an autosomal dominant trait, ${ }^{1-3}$ or as a syndromic component of osteogenesis imperfecta (OI). ${ }^{4}$ In this latter condition dental changes are overshadowed by frequent fractures and other variable manifestations, such as long bone deformity, blue sclerae, and deafness. Wormian bones in the cranial sutures are a consistent radiological finding in OI.

A kindred with autosomal dominant inheritance of DI, together with blue sclerae and wormian bones, has been investigated in South Africa. No previously published reports of this triad could be found. Of the 20 affected members of the family, a young man had the additional feature of skeletal fragility and on this basis the condition could be regarded as yet another example of heterogeneity within the general category of OI.

Delineation is of importance from the point of view of prognostication and genetic counselling and for these reasons the manifestations of the disorder are depicted and discussed in this paper.

Received for publication 4 June 1980

\section{The kindred}

The proband, a girl of mixed ancestry (IV $\cdot 20 ;$ fig 1 ), was referred to the Genetic Clinic at the age of 3 with a tentative diagnosis of OI. There was no history of fractures but she was found to have dentinogenesis imperfecta and blue sclerae, and skull radiographs showed multiple wormian bones in the cranial sutures. A positive family history was obtained and all available members of the kindred were examined clinically and radiographically. In a few patients, audiometry was undertaken and teeth were studied histologically and by electronmicroscopy.

The condition was diagnosed in 11 males and nine females in three generations of the kindred, while a positive history was obtained for nine others who were living abroad. The proband's grandfather and an affected uncle died after the survey had started and the latter's own offspring were not available for investigation. The pattern of transmission in the family was consistent with autosomal dominant inheritance (fig 1).

\section{Manifestations of the condition}

DENTINOGENESIS IMPERFECTA

The teeth had the yellow-brown discolouration which 


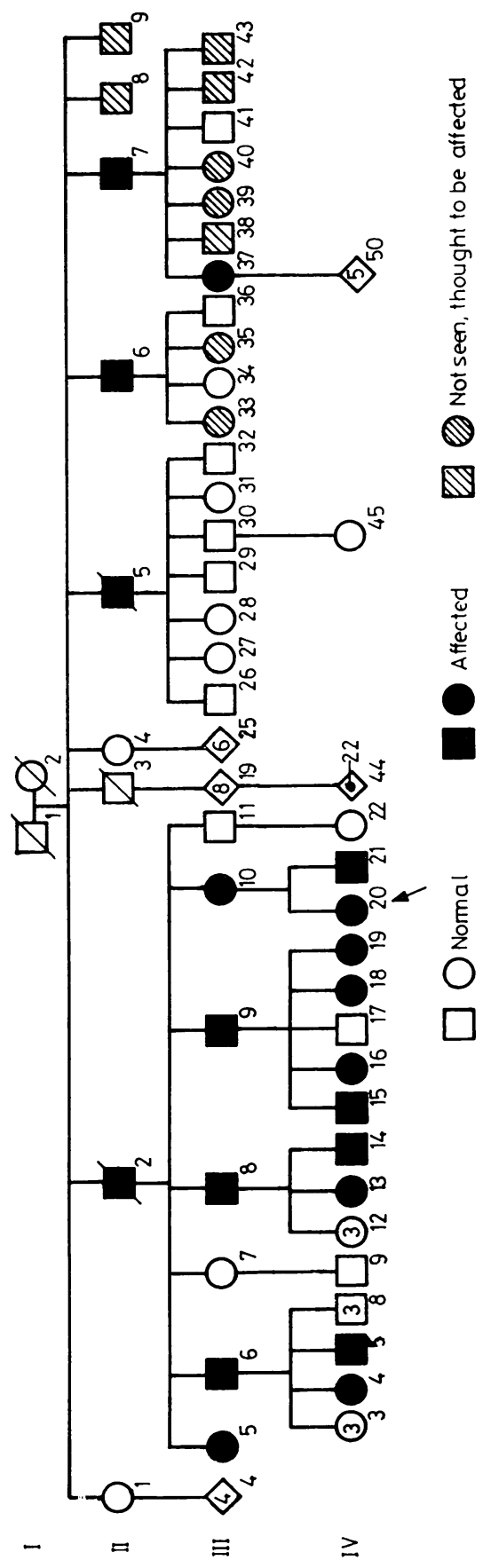

is characteristic of DI and were generally carious (fig 2). The edentulous affected members of the kindred all gave a firm history of teeth which had been pearly or opalescent and which had been extracted in childhood for severe caries.

\section{BLUE SCLERAE}

The sclerae were bluish-grey in all affected members of the kindred. The range of variation was not great and there was rarely confusion with normality, but the fact that the family were dark-skinned may well have influenced the actual tone of the scleral colour.

SKELETAL CHANGES

Lateral radiographs of the skull were obtained in nine affected subjects and multiple wormian bones were present in the cranial suture in each instance (fig 3). The degree of involvement was neither agenor sex-related and there were no problems of overlap with normality. In addition, radiolucent areas of

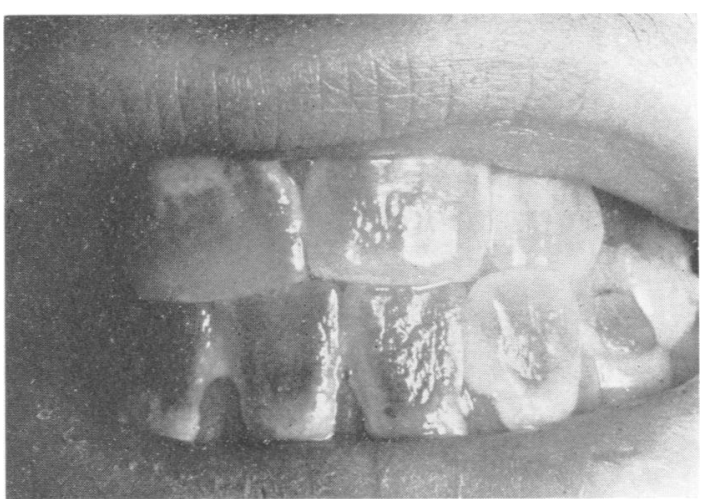

FIG 2 The teeth of an adolescent male $(I V \cdot 15)$ showing the opalescent discolouration that is characteristic of dentinogenesis imperfecta.

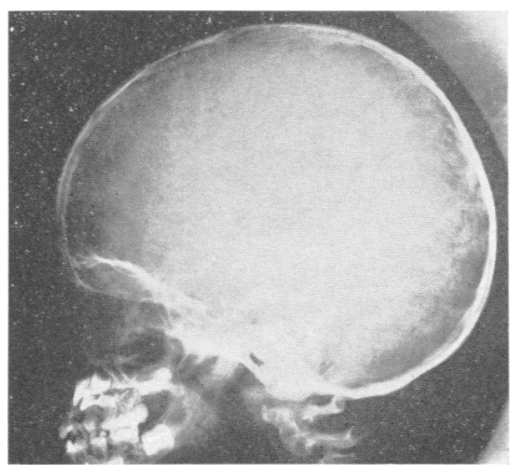

FIG 3 Lateral radiograph of the skull of a young girl $(I V \cdot 20)$ showing multiple wormian bones. 
osteoporosis circumscripta of variable extent were evident in the calvaria of six of these persons (fig 4).

Skeletal survey in six subjects showed generalised osteoporosis of moderate degree and in the older adults mild vertebral flattening and biconcavity had developed (fig 5). The weight bearing long bones, notably the femora, showed a moderate tendency to bowing, with pronounced diaphyseal cortical buttressing. In a male aged 72 years (II $\cdot 2)$ multiple small

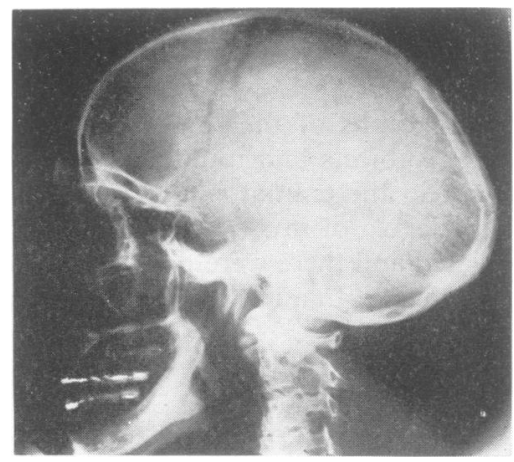

FIG 4 Lateral radiograph of the skull of the affected mother of IV 20 . Multiple wormian bones and areas of osteoporosis circumscripta are evident.

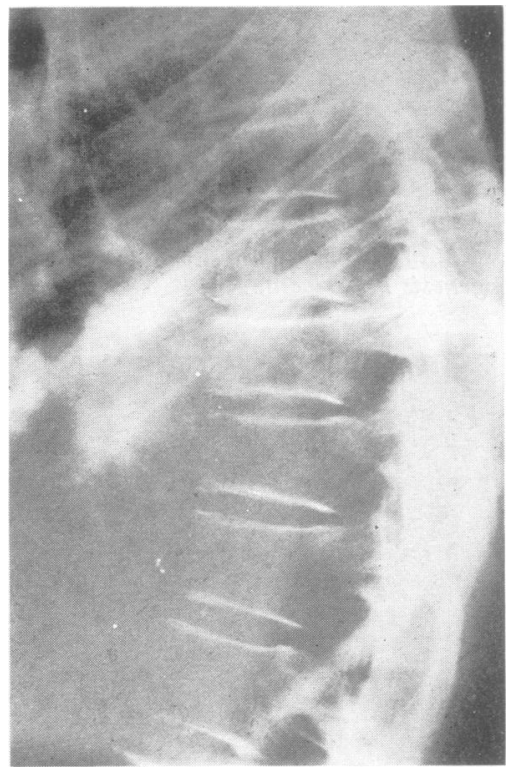

FIG 5 Lateral radiograph of the thoracolumbar spine of a male aged 72 years $(I \cdot 2)$. The vertebral bodies are porotic, with mild flattening and biconcavity. lucencies were present in the hyperostotic femoral cortices (fig 6). Dr R Murray of the Institute of Orthopaedics, Royal Orthopaedic Hospital, London interpreted these changes as "stress infarctions which might represent a minimal manifestation of osteogenesis imperfecta". Dr Murray commented that neither he nor other experts whom he had consulted had previously encountered similar changes.

Bony fragility was present in only one member of the kindred, an adolescent boy with pronounced platybasia, who had experienced ten femoral fractures on mild trauma (IV-15). None of the others had any tendency to undue fracturing and it must be assumed that this feature represents the end of the spectrum of severity of expression of the disorder.

\section{DEAFNESS}

The proband (IV-20) developed mild hearing impairment during early childhood and audiometric studies revealed equal bilateral perceptive hearing loss, which was worse with low tones. Her affected mother (III-10) also had episodic unilateral deafness, but it is uncertain whether these symptoms in either patient were directly related to the primary disorder. No other family members had hearing problems.

GENERAL FEATURES

The affected members of the kindred were of normal stature and, apart from the scleral and dental changes, their appearance was unremarkable. Intelligence was normal and there were no systemic ramifications. In particular, digital laxity and dermal

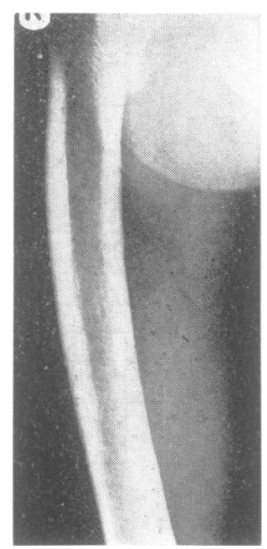

FIG 6 Anteroposterior radiograph of the shaft of the right femur of $I \cdot 2$. Moderate lateral bowing is evident and multiple small lucencies are present in the hyperostotic cortices. 
thinning or hyperextensibility, which are present in some form of OI, were not encountered in any of the respondents.

\section{LABORATORY FINDINGS}

Routine biochemical, haematological, and cytogenetic investigations yielded normal results in two children and three adults. In particular, the serum calcium, phosphorus, and alkaline phosphatase concentrations were consistently normal.

Histological and electronmicroscopical studies of an exfoliated lower deciduous incisor from the severely affected boy (IV - 15) were undertaken at the Dental Research Unit of the South African Medical Research Council, Johannesburg by Professor D H Retief. The following changes were reported: "Enamel was absent in incisal tip and on the labial surface but intact on the medial and lateral surfaces. Histologically, the tubular arrangement in the dentine was irregular and there was great reduction in the number of dentinal tubules when compared to a normal tooth. Scanning electronmicroscopy did not reveal any significant changes. The dentine surface was featureless but a space which was clearly visible at the enamel/dentinal junction was judged to be normal. The wide spacing of the dentinal tubules was confirmed".

\section{Discussion}

Dentinogenesis imperfecta may occur as an isolated autosomal dominant entity or as a component of some forms of OI. However, the triad of dentinogenesis imperfecta, blue sclerae, and wormian bones, as encountered in the family under review, does not seem to have been previously reported. McKusick (1979, personal communication) suggested that this entity should be regarded as a form of OI and it has been presented in this context.

Heterogeneity in OI has been recognised at both a clinical and a laboratory level and, although precise delineation of the sub-types has not yet been achieved, there is little doubt that there are several distinct forms of the condition. ${ }^{5}$ Tooth abnormalities may serve as a diagnostic indicator and this observation lends additional significance to the description and delineation of the syndrome under review.

The affected boy who had experienced several femoral fractures was the only member of the kindred to have any evidence of bone fragility. If he had been encountered as a sporadic case, a firm diagnosis of typical osteogenesis imperfecta would have been made, but in the light of the family circumstances it is reasonable to assume that his manifestations represented the end of the spectrum of severity of phenotypic expression of the abnormal gene. This situation exemplifies the gross variability of expression which can occur in this group of disorders and which causes difficulty during attempts at clinical delineation. It may be foreseen that biochemical characterisation will eventually provide the solution to the problem.

From the practical point of view, dental problems are the only important complication in the syndrome. Long bone bowing and vertebral flattening are of minor degree and radiographically apparent changes in the skull, notably wormian bones and osteoporosis circumscripta, do not produce clinical symptoms. It is uncertain whether the deafness in the two members of the kindred was causally related to the underlying condition. As previously mentioned, only one person had an undue tendency to fractures and on this basis bone fragility must be regarded as a very infrequent manifestation.

From the genetic standpoint the condition was transmitted as an autosomal dominant trait. The affected kindred were of mixed ancestry with genetic endowment from Malay, African Negro, European, and Xhoisan (Hottentot and Bushman) sources, but it was not possible to attribute any particular ethnic origin to the gene. As the disorder is biologically innocuous, fitness to reproduce is not compromised to any significant extent and it is possible that the gene had been transmitted through a long line of progenitors. If this is indeed the case families with the syndrome may ultimately be identified in diverse ethnic groups in other parts of the world.

I am grateful to Miss L Solomon and Mrs B Breytenbach for typing the manuscript and to Mrs G Beighton for preparing the pedigree, to Genetic Nursing Sisters Lecia Durr and Rosemary Duggan for assisting in the family studies, and to $\mathrm{Mr} \mathrm{A} \mathrm{A}$ de Méneaud for preparing the illustrations.

I am also grateful to Professor V A McKusick for his helpful comments on the syndromic status of this disorder, to Professor D H Retief for the histological studies of the teeth, and to Dr R O Murray for his helpful opinion on the radiographic changes.

This project was supported by grants from the South African Medical Research Council and the University of Cape Town Staff Research Fund.

\footnotetext{
References

1 Johnson ON, Chaudhry AP, Gorlin RJ, Mitchell DF, Bartholdi WI. Hereditary dentınogenesis imperfecta. J Pediatr 1959;54:786-92.

2 Shokeir MHK. Dentinogenesis imperfecta: severe
} 
expression in a prooable heterozygote. Clin Genet 1972; 3:442-7.

3 Miller WA, Winkler S, Rosenberg JJ, Mastracola R, Fischman SL, Wolfe RJ. Dentinogenesis imperfecta traceable through five generations of a part American Indian family. Oral Surg 1973;35:180-6.

4 McKusick VA. Heritable disorders of connective tissue. St Louis: Mosby, 1972:390.
5 Sillence, DO, Senn A, Danks DM. Genetic heterogeneity in osteogenesis imperfecta. J Med Genet 1979;16:101-16.

Requests for reprints to Professor $\mathbf{P}$ Beighton, Department of Hùman Genetics, The Medical School, University of Cape Town, Observatory 7925, South Africa. 